\title{
Resiliencia y juicio moral, factores fomentadores de conductas y actitudes de liderazgo personal y social
}

\section{Resilience and moral judgment, factors that encourage behaviors and attitudes of personal and social leadership}

\author{
David Alberto Zamudio Flores ${ }^{1}$
}

${ }^{1}$ Facultad de Humanidades, Universidad Continental, Huancayo

\section{RESUMEN}

El objetivo del presente trabajo es explicar y prestar especial atención a dos temas que son la resiliencia y el juicio moral, los cuales son factores que influyen especialmente a desarrollar conductas y actitudes de liderazgo, cada uno de ellos desarrollado en un alto nivel y complementándose pueden ejercer en las personas desde muy temprana edad un correcto entendimiento y accionar en base a forjarse como líderes a nivel personal y social, lo cual contribuya a la sociedad influenciando e inspirando un verdadero liderazgo. El tema de liderazgo se trata y estudia desde temprana edad, pero en muchas ocasiones queda solo en un tema teórico y si se trata de abordarlo de manera práctica, no siempre es de la mejor manera puesto que si se cree que solo realizando dinámicas o ejercicios de competencia se estimula liderazgo se está perdiendo el objetivo, se tiene que buscar y desarrollar un cambio interno, un desarrollo personal que esté basado en enfrentar y superar las dificultades y adversidades de la vida de manera sana, positiva y valiente, y que no perdamos de vista la gran importancia de ejercer comportamientos ligados con la ética, con la importancia que se le debe dar y practicar los principios éticos que fundamenten nuestras conducta de un buen liderazgo, empático y sensible hacia los demás. Todo ello está relacionado con la práctica y desarrollo de la resiliencia y el juicio moral, factores que se abordan en el presente trabajo y que contribuyen a la salud mental, y sobremanera al desarrollo del liderazgo personal y social.

Palabras clave: Resiliencia, juicio moral, influencia, liderazgo.

\begin{abstract}
The objective of this paper is to explain and focus special attention in two issues that are resilience and moral judgment, which are factors that influence especially develop behaviors and attitudes of leadership, each of them developed at high level and complementing each other to perform in people from an early age a correct understanding and to act on the basis of forging themselves as leader a personal and social level, which contributes influencing and inspiring true leadership in the society. The topic of leadership is treated and studied from an early age, but in many cases it remains only in a theoretical topic and if it is dealt with in a practical way, it is not always in the best way since it is believed that only by performing dynamics or exercises of competition, leadership is stimulated, the objective is being lost, we must seek and develop an internal change, a personal development that is based on facing and overcoming the difficulties and adversities of life in healthy, positive and courageous way, and that we do not lose of view the great importance of exercising behaviors linked to ethics, with the importance that should be given and practiced the ethical principles that underlie our conduct of good leadership, empathetic and sensitive towards others. All this is related to the practice and development of resilience and moral judgment, factors that are addressed in the present work and that contribute to mental health, and greatly to the development of personal and social leadership.
\end{abstract}

Keywords: Resilience, moral judgment, influence, leadership.

Historial del artículo:

Recibido, 22 de febrero de 2017; aceptado, 10 de junio de 2017; disponible en línea, 25 de junio de 2017

* Psicólogo, docente de la Universidad Continental.

Correo: dzamudio@continental.edu.pe 


\section{INTRODUCCIÓN}

Es indudable la importancia que tiene el liderazgo en la vida de toda persona, y así también, el gran efecto que genera la aparición o surgimiento de un líder en un equipo, sociedad o nación puesto que influirá significativamente en la manera de pensar y comportarse de los demás.

La curiosidad por el tema del liderazgo no es algo propio de nuestros días, sino que ha sido ampliamente estudiado, y en especial desde el campo de la psicología social, así como de la psicología del trabajo y de las organizaciones y de ciencias afines (Palomo, 2013). Es por ello que, el liderazgo es un tema debatible y ha sido y será de gran relevancia siempre en todo ámbito y contexto, puesto que llegar a ejercer un verdadero liderazgo no es una labor sencilla requiere de mucha valentía, constancia y coherencia, pero así también se reconoce, valora y respeta imperecederamente a la persona que llega a desarrollar e inspirar un verdadero liderazgo.

Acertadamente Bennis (1959), citado por Palomo (2013, p. 21), menciona que: "De entre todas las áreas oscuras y confusas en psicología social, la del liderazgo lucha indudablemente por alcanzar el primer puesto. E, irónicamente, probablemente sea el liderazgo más que cualquier otro tópico en ciencias sociales, el tema del que más se ha escrito, pero sobre el que menos se conoce". Es así que una interrogante que surge es, que si bien podemos definir el liderazgo, ¿Qué acciones debemos alentar y promover para que pueda surgir y desarrollarse en un equipo y/o sociedad?

\section{Influencia y liderazgo}

Según Lussier y Achua (2011) definen el liderazgo como: "El proceso de influencia entre líderes y seguidores para lograr objetivos organizacionales por medio del cambio". Es así que la influencia es un elemento clave en el liderazgo, puesto que a través de la capacidad que se posea al influir va delimitar en gran medida el éxito y la trascendencia de un líder para lograr un objetivo determinado, es decir, una meta compartida conjuntamente con sus seguidores.

Worchel et al (2002) mencionan: "En cualquier grupo, grande o pequeño, algunos individuos tienen más poder e influencia que los otros. Quien tiene esta influencia mayor asume una posición de importancia en el grupo y tiende a ser reconocido como el líder".

Enseñar por medio del ejemplo es una de las principales manera de influir por parte de un líder ya que no solo queda en palabras lo que transmite, propone, o quiere enseñar, sino que también lo evidencia, lo proyecta, lo ejerce.

\section{Influencia a partir de la resiliencia}

Ser resiliente es una acertada forma de enseñar e influenciar a través del ejemplo a ser líderes. Resiliencia se define según Lamas (2002) como la capacidad de los seres humanos para sobreponerse a las crisis y construir positivamente sobre ellas, basada en unos factores que facilitan y amplifican dicha respuesta. En otras palabras, es el sistema que se desarrolla por parte de un individuo o un grupo para enfrentar efectivamente la adversidad. Vanistendael (1994) la define como la capacidad del ser humano o de un sistema social de vivir bien y desarrollarse positivamente a pesar de las condiciones de vida difíciles. Esto implica una capacidad de resistencia y una facultad de construcción positiva. Para Grotberg (1996), citado en Panez et al (2000, p16), define la resiliencia como: "La capacidad humana universal para hacer frente a las adversidades de la vida, superarlas e incluso ser transformadas por ellas. La resiliencia es parte del proceso evolutivo y debe ser promovida desde la niñez". Silva (1999), citado en Panez et al (2000, p16), la define como "la capacidad universal de todo ser humano de resistir ante condiciones adversas y recuperarse, desarrollando paulatinamente respuestas orientadas hacia la construcción de un ajuste psicosocial positivo al entorno, a pesar de la existencia simultánea de dolor y conflicto intrapsíquico".

American Psychological Association (APA) (2011) define la resiliencia como: "El proceso de adaptarse bien a la adversidad, a un trauma, tragedia, amenaza, o fuentes de tensión significativas, como problemas familiares o de relaciones personales, problemas serios de salud o situaciones estresantes del trabajo o financieras", así también enfatiza que "ser resiliente no quiere decir que la persona no experimenta dificultades $\circ$ angustias e incluye conductas, pensamientos y acciones que pueden ser aprendidas y desarrolladas por cualquier persona".

La resiliencia es una capacidad universal, que permite a una persona, grupo o comunidad, minimizar o sobreponerse a los efectos nocivos de la adversidad, puede transformar o fortalecer la vida de la persona para Kotliarenco et al (1996). Es así que se entiende que la resiliencia es el afrontamiento, aprendizaje y superación personal a raíz de una dificultad o crisis personal y/o social, permite que no solo desarrollemos salud mental sino que también a través de nuestras actitudes y pensamientos al momento de enfrentar adversidades o dificultades lo podamos realizar de manera positiva y valiente, es decir, siendo líderes en nuestras vidas.

El liderazgo entonces está directamente relacionado con cuan resiliente una persona es, esta capacidad de afrontar las dificultades, superarlas y salir fortalecido de ellas se ve representada al ejercer los diez rasgos 
de los lideres efectivos propuestos por Lussier y Achua (2011), los cuales son:

a) Dominio, la cual es la disposición que ejerce una persona por asumir una responsabilidad a través de estar a cargo de un equipo, para lo cual requiere que haya generado una capacidad de sobreponerse a las dificultades a través de la resiliencia de tal manera que pueda seguir generando esa valentía por asumir la responsabilidad de liderar un equipo.

b) Gran energía, que es la capacidad que tiene una persona de no dejarse abatir y tener presente una motivación intrínseca que en gran medida son características de una persona resiliente que no se rinde o amilana ante las dificultades.

c) Confianza personal, la cual se logra a través de la autoestima que es el grado de valoración personal que tiene una persona sobre sí misma, y que si ésta es alta podrá ejercer la resiliencia de manera natural.

d) Locus de control internalizador, que es tomar la responsabilidad de nuestras propias vidas, es decir, no buscar justificaciones o excusas ante situaciones poco favorables, sino sobreponerse a las dificultades y salir fortalecido, lo cual evidencia resiliencia.

e) Estabilidad, que es la capacidad que posee una persona para identificar y reconocer sus cualidades y defectos, lo cual permita encontrar el equilibrio y sosiego necesario para buscar alternativas de solución ante dificultades o crisis.

f) Integridad, que es la práctica de valores y que se relaciona con la resiliencia en como superamos las dificultades de manera positiva, sin dañar a otras personas, sin transgredir leyes o faltar el respeto a otros en un afán de superar crisis o dificultades.

g) Inteligencia, que es la capacidad de resolver problemas novedosos de manera creativa, característica del accionar de una persona resiliente ante dificultades o crisis puesto que busca acciones y concesiones como estrategias de superación.

h) Inteligencia emocional, la cual se define como la capacidad de identificar, reconocer y manejar nuestras emociones y las emociones de las demás personas, y que tiene una importante función al momento de buscar alternativas de solución ante crisis o dificultades.

i) Flexibilidad, que es la disposición que tiene una persona a no amilanarse ante situaciones nuevas, sino ser consciente de la oportunidad de aprender de nuevos desafíos o nuevas experiencias, es decir la valentía de encarar nuevas experiencias, traigan éstas éxitos o fracasos, lo cual fortalece nuestra resiliencia; y como último rasgo.

i) Sensibilidad hacia lo demás, que es la práctica de la empatía y asertividad para con otras personas, características esenciales de la resiliencia que involucra enfrentarse, superar y salir fortalecido de las dificultades de la vida pero sin atentar en contra de los derechos y el respeto hacia de otras personas, es decir que se ejerza de manera positiva. Grotberg (1996) menciona que la resiliencia no solo es efectiva para enfrentar adversidades sino también para la promoción de la salud mental y emocional de las personas. Es así que al fomentar y trabajar por el desarrollo de la resiliencia no solo se está desarrollando salud mental, añadido a ello también se está promoviendo conductas y actitudes de liderazgo entre las personas, seguidores, en un equipo y en la sociedad.

\section{Influencia a partir del juicio moral}

Otro medio acertado de enseñar e influir a través del ejemplo a ser líderes, es a través de tener un comportamiento ligado a la ética, es decir creer y practicar valores y principios éticos sociales, lo cual es desarrollado por el juicio moral, el cual se define como aquel acto mental que afirma o niega el valor moral ante una situación determinada o un comportamiento del que somos testigos, es decir, el juicio moral se pronuncia sobre la presencia o ausencia de una valor ético, en una situación o comportamiento concreto (Definición ABC, 2017).

Reboiras et al (1995), entienden por Juicio Moral, a la capacidad que todo sujeto tiene para establecer proposiciones ético-valorativas acerca de hechos de la realidad que tienen que ver con las conductas propias o semejantes. Así mismo, la existencia del juicio moral hace posible, en la persona que lo posee, regular sus interacciones con otros, de acuerdo con ciertas normas que él ha construido o adoptado. Además, afirma que; en todo sujeto hay una estructura normativa que es la mediadora en la relación con la realidad.

Piaget (1967) y Kohlberg (1978), son los dos investigadores más importantes sobre este tema, desde su perspectiva psicológica ellos señalan que el juicio moral se desarrolla a través de un proceso racional que es paralelo al desarrollo cognoscitivo.

Piaget (1984), menciona que estudiar el desarrollo del juicio moral es adentrarse en el ámbito cognitivo, según el autor la construcción de los procesos del conocimiento avanzado, se desarrolla paralelamente a la construcción de los principios éticos.

Hersh et al (1984), manifiestan que Jean Piaget comenzó a desarrollar su estudio sobre juicio moral al estudiar este tema en los niños, todo ello como parte de su interés por entender como los niños se orientan a la sociedad, fue influenciado por el Sociólogo francés Durkheim, quien postulaba que lo más importante de la educación moral era enseñar a los niños a dedicarse al bien de la sociedad y ceñirse a la obediencia a las reglas morales de la misma, desarrollando un sentido 
de solidaridad y el respeto por las reglas.

Hersh et al (1984), señalan que Kohlberg (1978), inspirado por Piaget, desde 1978, se propuso estudiar la evolución del desarrollo moral en los seres humanos, es así que el juicio moral es la capacidad de un sujeto de ponerse en el lugar del otro según Kohlberg, es así que no es un simple adaptación del sujeto al medio sobre el que actúa, sino que se desarrolla en ámbitos de relaciones interpersonales, es así que el sujeto sea capaz de verse a sí mismo como si fuera la persona que tiene al frente, de imaginar qué pensaría si estuviera en las circunstancias ajenas, es decir siendo empático.

Mira y López (1972), refiere que el juicio moral es un producto histórico y social, por tal razón las personas se encuentran en proceso de desarrollo y cambios, por ende los propios valores se encuentran en constante cambio, se modifican, es así que entonces la moral no es estática ni uniforme.

Kohlberg et al (1989), refieren que a la persona se le puede orientar para que desarrolle su juicio moral, no predicándole o moralizándole, tampoco con castigos o recompensas, sino aplicando el uso de dilemas morales los cuales tengan un grado de dificultad, y ayudándole a discernir y elaborar sus pensamientos y respuestas en relación a ellos.

Frisancho (2001), manifiesta que Lawrence Kohlberg presenta 3 estadios bien definidos en relación al desarrollo del juicio moral, y que son los siguientes:

a) Nivel Pre - Convencional.- En este nivel la determinación de lo que es bueno o malo, las normas o reglas se respetan en función de las consecuencias (premio o castigo) o el poder de quienes las establecen. Se juzga en función de las consecuencias que se obtienen de las acciones que se realiza, no se comprende aún que las normas sociales son necesarias para un buen funcionamiento de la sociedad.

b) Nivel Convencional.- En este nivel las personas viven identificados con los pares, con el grupo. Los problemas morales son enfocados desde la perspectiva de ser un miembro de la sociedad, tomándose en consideración al grupo, aquí el castigo o recompensa pierde importancia puesto que los juicios de lo que es bueno o malo se basan en lo que el grupo o la sociedad así lo consideran o establecen.

c) Nivel Post-Convencional.- En este nivel las personas enfocan los problemas morales desde una perspectiva que trasciende al grupo social. Aquí los juicios, razonamientos y las decisiones están determinados en base a los principios morales los cuales tienen una validez universal y pesan más que las normas o reglas establecidas por un grupo y que pueden estar equivocadas.
Es así que un líder desarrolla coherencia entre lo que piensa, dice y hace, todo ello enmarcado en valores, influencia e inspira esa coherencia entre sus seguidores, lo cual es una tarea de por sí difícil pero admirable, característico de un líder, consecuentemente ello está relacionado con la integridad y sensibilidad hacia los demás, dos de los diez rasgos de líderes efectivos que nos mencionan Lussier y Achua (2011), y que se detallaron anteriormente. Es por ello que el liderazgo influye e inspira actitudes y acciones que propicien y desarrollen beneficios y resultados positivos al equipo, a la sociedad, a partir de un objetivo en particular, o una meta compartida, los cuales se logran de manera genuina a través de ejercer empatía y la práctica de valores tales como la responsabilidad, la honestidad, la puntualidad, la gratitud, el respeto, entre otros y evidentemente no está involucrado con acciones egoístas o individualistas que estén relacionadas con antivalores los cuales propicien caos e inestabilidad en un equipo o en una sociedad.

\section{Resiliencia y juicio moral en el liderazgo}

Es por ello que la resiliencia y el juicio moral, factores fomentadores de conductas y actitudes de liderazgo personal y social contribuyen de sobremanera en ejercer un verdadero liderazgo, influyente, inspirador y coherente, un liderazgo que permita y contribuya con un desarrollo a nivel individual y en equipo, no solo por parte del líder sino también por parte de los seguidores, es decir, liderazgo social.

Si bien estos dos factores son de suma importancia en relación a fomentar actitudes y conductas de liderazgo en base a la influencia, una pregunta importante que surge es cuál es la relación entre los dos, es decir, $\grave{2}$ Las personas que tienen un mayor nivel de resiliencia tendrán un mayor o un menor nivel de juicio moral?, ¿̇Existe una relación directa o inversa entre ambas variables?, si bien la resiliencia como se detalló anteriormente es la capacidad de una persona o sistema social de enfrentar adecuadamente las dificultades, de una "forma socialmente aceptable", lo cual da a entender que la resiliencia es ejercida por una persona que ha desarrollado claramente su juicio moral hasta el segundo nivel según Kohlberg, es decir, el nivel convencional, pero, no necesariamente habrá llegado a desarrollar hasta el nivel post convencional, entonces, una persona que es altamente resiliente no necesariamente tiene un alto nivel de juicio moral, puesto que enfrentará y superará las dificultades de una forma socialmente aceptable, debido a que puede tener la fortaleza pero no necesariamente la conciencia que le permita reflexionar, puede que su accionar resiliente se sustente a través de patrones culturales de respeto al prójimo, en relación a lo que la sociedad considera y establece como reglas sociales, en base a normas, autoridad y expectativas 
del grupo, pero que no tenga un elevado nivel de juicio moral que realmente lo haga discernir y juzgar de manera objetiva y valiente entre lo bueno o malo en determinadas situaciones, es decir que luche y crea en principios éticos racionalmente escogidos desde una perspectiva que trascienda al grupo social.

Así mismo, una persona que ha desarrollado un alto nivel de juicio moral no necesariamente será una persona que haya desarrollado un alto nivel de resiliencia, puesto que puede haber alcanzado un alto nivel de juicio moral o haber llegado a desarrollar en el mejor de los casos hasta el nivel post convencional, por el cual tiene la facultad de anteponer y luchar por principios éticos antes que por leyes y reglas acordadas por los pares o la sociedad, pero que no pueda enfrentar, superar, y sobreponerse a las adversidades o dificultades de la vida de manera sana y positiva.

Es crucial identificar cual es la relación entre estos dos factores para tomar medidas debidamente elaboradas que permitan que ambos factores se puedan desarrollar y complementar, es decir, que entre ambos se trabajen en consonancia, propiciando una sinergia que se desarrollen en las personas y en los equipos con congruencia y que sean "catalizadores" en el desarrollo entre ambos factores, los cuales favorezcan el desarrollo de conductas y actitudes de liderazgo.

\section{CONCLUSIONES}

Tanto la resiliencia como el juicio moral son dos temas cruciales los cuales están ligados directamente con el liderazgo, no solo lo fomentan sino que también, y principalmente, acompañan durante la formación de un líder y así también en la formación de líderes entre sus seguidores, es decir en la influencia que ejercen. Conocer y comprender la importancia de estos dos temas, y así también investigar sobre la relación que existe entre ambos factores, permitirá desarrollar y fomentar conductas y actitudes de liderazgo personal y social, así también, en última instancia permitirá identificar y reconocer conductas y actitudes de liderazgo en los integrantes de un equipo o sociedad lo cual permita reconocer a quienes realmente tienen la capacidad de ser líderes; vale decir nuevamente que son factores que contribuyen de sobremanera no solo con el tema de liderazgo personal y social, sino que también con la salud mental de las personas y de los equipos.

\section{REFERENCIAS BIBLIOGRÁFICAS}

Palomo M. Liderazgo y motivación de equipos de trabajo. 8ª edición. Madrid: ESIC Editorial: 2013.
Bennis W. Leadership Theory and Administrative Behavior: The Problem of Authority. Administrative Science Quarterly; 1959: 259-260.

Lussier R, Achua C. Liderazgo: Teoría, aplicación y desarrollo de habilidades [Leadership: Theory, Aplicación, \& Skill Development]. 4a edición. México: Cengage Learning Editores; 2011.

Worchel S, Cooper J, R.Goethals G, M.Olson J. Psicología Social. Estados Unidos: International Thomson Editores; 2002.

Lamas H. Educar para la competencia personal. Palabra de maestro. 2002; 11 (35): 44- 47.

Vanistendael S. Resilience: a few keys issues. Malta. International Catholic Child Bureau; 1994.

Grotberg E. Guía de promoción de la resiliencia en los niños para fortalecer el espíritu humano. La Haya (Países Bajos): Editorial Bernard van Leer Foundation; 1996.

Panez R., Silva G. Silva M. Resiliencia en el ande: un modelo para promoverla en los niños. Lima: P\&S Ediciones; 2000.

Silva G. Resiliencia y violencia política en niños. Buenos Aires: Universidad Nacional de Lanús, C.E.I.R.: Bernard van Leer Foundation; 1999.

American Psychological Association. El camino a la resiliencia [Internet]. Washington, DC: APA; 2011 Julio [Citado el 24 de Mayo de 2017). Disponible en: http://www.apa.org/centrodeapoyo/ resiliencia-camino.aspx

Kotliarenco M, Cáceres I, Álvarez C. Resiliencia. Construyendo en adversidad. Santiago: Centro de estudios y atención del niño y la mujer (CEANIM); 1996.

DefinicionABC. Definición de juicio moral. [Citado el 25 de Mayo de 2017). Disponible en: http://www. definicionabc.com/general/juicio-moral.php

Reboiras J, Bianchi A., Finardi M. La Adolescencia, Enfoque multidimensional, Docencia. Buenos Aires; 1995.

Piaget J. Los procedimientos de la educación moral. $3^{\text {a }}$ edición. Buenos Aires: Ed. Losada; 1967.

Kohlberg L. El niño como filósofo moral. Madrid: Alianza Editorial; 1978.

Piaget J. El criterio moral en el niño. 2 ${ }^{a}$ edición. Barcelona: Ed. Fontanella; 1984.

Hersh R, Reimer J, Paolitto D. El crecimiento moral. De Piaget a Kohlberg. Madrid: Editorial Narcea; 1984.

Mira y López E. Psicología evolutiva del niño y del

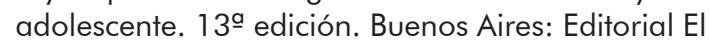
Ateneo; 1972.

Kohlberg L, Berkowitz M, Cañón C. El sentido de lo humano. Valores, psicología y educación. 2a edición. Bogotá: Biblioteca Internacional; 1989.

Frisancho S. Educación y desarrollo moral. Programa especial MECEP. Lima: Ministerio de Educación. GTZ Cooperación técnica República Federal de Alemania y KFW Cooperación Financiera República Federal de Alemania; 2001. 